\title{
Estimation of Aperture of the Tunka-Rex Radio Array for Cosmic-Ray Air-Shower Measurements
}

\section{Vladimir Lenok ${ }^{a, *}$ on behalf of the Tunka-Rex Collaboration}

(a complete list of authors can be found at the end of the proceedings)

\author{
${ }^{a}$ Karlsruhe Institute of Technology, Institute for Astroparticle Physics, D-76021 Karlsruhe, Germany \\ E-mail: contact@tunkarex.info
}

\begin{abstract}
The recent progress in the radio detection technique for air showers paves the path to future cosmic-ray radio detectors. Digital radio arrays allow for a measurement of the air-shower energy and depth of its maximum with a resolution comparable to those of the leading optical detection methods. One of the remaining challenges regarding cosmic-ray radio instrumentation is an accurate estimation of their efficiency and aperture. We present a probabilistic model to address this challenge. We use the model to estimate the efficiency and aperture of the Tunka-Rex radio array. The basis of the model is a parametrization of the radio footprint and a probabilistic treatment of the detection process on both the antenna and array levels. In this way, we can estimate the detection efficiency for air showers as function of their arrival direction, energy, and impact point on the ground. In addition, the transparent internal relationships between the different stages of the air-shower detection process in our probabilistic approach enable to estimate the uncertainty of the efficiency and, consequently, of the aperture of radio arrays. The details of the model will be presented in the contribution.
\end{abstract}

$37^{\text {th }}$ International Cosmic Ray Conference (ICRC 2021)

July 12th - 23rd, 2021

Online - Berlin, Germany

\footnotetext{
${ }^{*}$ Presenter
} 


\section{Introduction}

Aperture estimation for a cosmic-ray radio antenna array is an open problem in modern groundbased observational astroparticle physics. This work presents a probabilistic model of the detection efficiency combined with a semi-analytic way of the aperture estimation, which both form the aperture model. The model was developed for the Tunka-Rex instrument; however, it can be applied to other cosmic-ray radio instruments.

\subsection{Tunka-Rex Instrument}

The Tunka Radio Extension (Tunka-Rex) is a cosmic-ray digital radio antenna array located at the site of the TAIGA facility in the Tunka valley in Siberia [1,2]. The antenna array evolved and consisted of 63 short aperiodic loaded loop antennas (SALLA [3]) distributed over about $1 \mathrm{~km}^{2}$ by the operation's end in 2019. The antennas' operating frequency band ranges from 30 to $80 \mathrm{MHz}$.

\subsection{Definition of Aperture}

Aperture as a physical quantity naturally appears in consideration of the number, $N$, of cosmic rays observed by an instrument. This number equals the cosmic-ray flux $J$ multiplied to exposure $\epsilon$ of the instrument, namely, $N=\epsilon J$. The exposure is the crucial factor for the reconstruction of the cosmic-ray flux's characteristics from the observations. Its generic form can be expressed as the integral of the efficiency $\xi$ over the area of fiducial angular selection, $\Omega_{f}$, the instrumental fiducial area, $S_{f}$, and the duration of the measurements, $T$

$$
\epsilon=\int_{T} \int_{\Omega_{f}} \int_{S_{f}} \xi \cos \theta d s d o d t
$$

If the efficiency does not constantly change, the time-independent part of the integral can be considered separately. This part is referred to as "aperture"

$$
A=\int_{\Omega_{f}} \int_{S_{f}} \xi \cos \theta d s d o .
$$

This integral can be transformed

$$
\begin{aligned}
\int_{\Omega_{f}} \int_{S_{f}} \xi \cos \theta d s d o & =S_{f} \int_{\Omega_{f}} \int_{S_{f}} \frac{\xi}{S_{f}} \cos \theta d s d o= \\
& S_{f} \int_{\Omega_{f}}\left(\frac{1}{S_{f}} \int_{S_{f}} \xi d s\right) \cos \theta d o=S_{f} \int_{\Omega_{f}}\langle\xi\rangle_{s} \cos \theta d o=S_{f} A_{\Omega} .
\end{aligned}
$$

The symbol $\langle\xi\rangle_{S}$ denotes the averaged efficiency over the fiducial area. The last integral denoted as $A_{\Omega}$ is referred to as "angular aperture" ${ }^{1}$. Thus, by collecting the introduced quantities, the exposure estimation can be expressed in the factorized form

$$
\epsilon=S_{f} A_{\Omega} T
$$

\footnotetext{
${ }^{1}$ In the same fashion, the aperture could be factorized into a solid angle of the fiducial angular selection and the averaged efficiency over this angle. Even though this approach can have advantages in some studies, since it preserves the angular coverage of the instrument in case of selection of the full-efficiency regions, it is out of the scope of the present work.
} 


\section{Efficiency Model}

As it is clear from the definition above, efficiency is the critical component in aperture estimation. The usual approach of the efficiency estimation with extensive libraries of the Monte-Carlo simulations is cumbersome in studying air-shower radio emission since the corresponding simulations are computationally expensive. We use a different approach for the aperture study — we build a model for the instrument efficiency and use it in the aperture estimation.

The idea behind the model is to exploit the probabilistic properties of air-shower radio footprint characteristics and the properties of the air-shower detection process. The model consists of three components: the model of air-shower radio footprint (section 2.1), the model of signal detection on the level of individual antennas (section 2.2), and the model of entire-array triggering (section 2.3).

\subsection{Spatial Distribution of Radio Signals}

The Tunka-Rex asymmetric lateral distribution function in the discussed model is the means of predicting the spatial distribution of the electric field, $\mathscr{E}$, corresponding to a given set of the air-shower parameters, i.e., incoming direction, energy, $E$, depth of shower maximum, $X_{\max }$. The reconstruction uses the same function [4].

The model describes the distribution in the geomagnetic coordinate system, the system built on the basis of the shower propagation vector $\mathbf{V}$ and the local geomagnetic field $\mathbf{B}$ vector.

The lateral distribution function shows only the most probable value of the electric field. The distribution of the electric field strength for a given spatial point is an inseparable component of the model. We model this distribution with the normal distribution with a standard deviation of $14.47 \%$ of the field value centered on the most probable value. The value of the standard derivation comes from a direct comparison of the model predictions and the Monte-Carlo simulated signals.

\subsection{Signal Detection by Individual Antennas}

The process of signal detection by individual antennas has a probabilistic nature because of the unavoidable presence of noise. Even though this nature is especially pronounced for small signals, it significantly influences the detection process for a wide range of signals relevant for the air-shower radio array operation.

To describe this probabilistic behavior, we processed the Monte-Carlo generated electric field with the Tunka-Rex signal-processing pipeline [5], which includes the antenna model, response of the electronic components, and signal analysis procedure. The processing was done multiple times with different samples of the on-site measured noise samples. The fraction of times the standard analysis pipeline of Tunka-Rex could detect a given signal becomes the detection probability for this signal. The S-shaped curve parametrizes the obtained dependence of the detection probability $p_{0}$ as a function of the signal strength $S$

$$
p_{0}(S)=\frac{1}{2}+\frac{1}{2} \tanh \frac{S-S_{1 / 2}}{S_{0}^{\prime}+S_{0}^{\prime \prime} S} .
$$

Within the framework of the model, the detection probability is not a number but a random variable characterized by the probability density of the beta distribution form

$$
P=\frac{p^{\alpha-1}(1-p)^{\beta-1}}{\mathrm{~B}(\alpha, \beta)}
$$



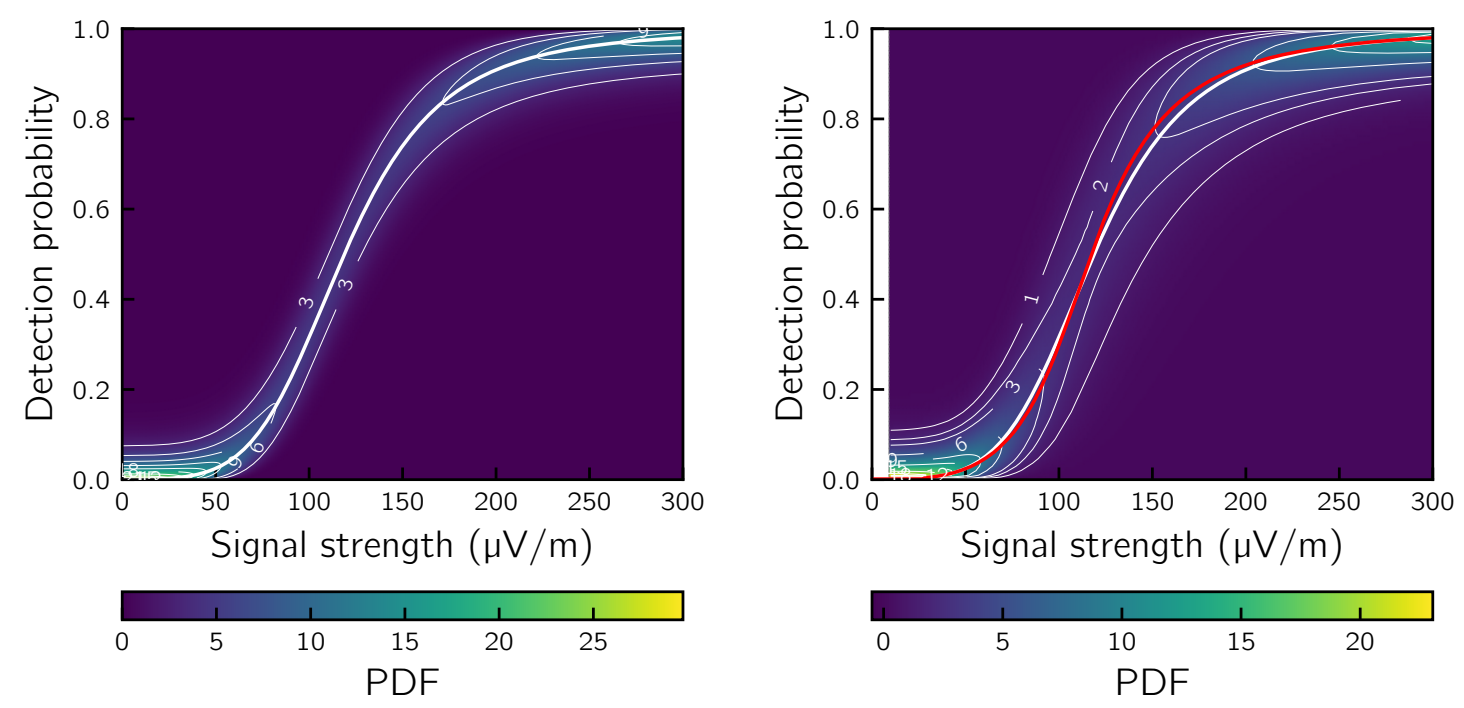

Figure 1: The probability density function of the signal detection on the individual antenna level. Left: initial function. The white line shows the most probable value. Right: the function after the marginalization procedure taking into account the uncertainty of the signal description. The white and red lines indicate the most probable values for the function before and after the marginalization.

with the parameters depending on the most probable value $\alpha=n p_{0}+1, \beta=n-n p_{0}+1$, and the number of repetitions of the signal processing, $n$, that equals 30 for the present work. The left plot in Figure 1 shows the obtained probability density.

To account for the uncertainty of the signal description, we apply a marginalization procedure. The function under the marginalization is a combination of the probability density of signal detection (6) and the probability density of observing a signal with a certain strength modeled with the normal distribution, $\mathcal{N}$, with the standard deviation equal to $14.47 \%$ found from the comparison of the model and the simulations, $\sigma_{S}=0.1447 \mathrm{~S}$. The combined density is subject to marginalization over the signal strength

$$
P_{S}(S)=\int_{0}^{\infty} \frac{p^{\alpha\left(S^{\prime}\right)-1}(1-p)^{\beta\left(S^{\prime}\right)-1}}{\mathrm{~B}\left(\alpha\left(S^{\prime}\right), \beta\left(S^{\prime}\right)\right)} \mathcal{N}\left(S, \sigma_{S}^{2}\left(S^{\prime}\right)\right) d S^{\prime}
$$

The marginalization makes the probability density wider, allowing for a broader range of the possible realizations of the detection probability. The right plot in Figure 1 shows the obtained marginalized function.

\subsection{Fulfillment of Trigger Condition}

This stage combines the probability densities obtained on the individual antenna level into a final probability density used to infer the detection probability value for a given shower. The entire Tunka-Rex array triggering happens when at least a certain number of antennas detect signals. Two methods can provide probability estimation for such triggering scheme. 
Probabilistic Calculations. One way to estimate the probability of observing a given number of signals over the antenna field is to perform the computation of probabilities of all possible situations not leading to the triggering and to subtract them from unity

$P=1-\sum_{i=1}^{\left(\begin{array}{c}N \\ 0\end{array}\right)} p_{i}^{(0)}-\sum_{i=1}^{\left(\begin{array}{c}N \\ 1\end{array}\right)} p_{i}^{(1)}-\cdots-\sum_{i=1}^{\left(\begin{array}{c}N \\ m-1\end{array}\right)} p_{i}^{(m-1)}$

Each term is a sum of all possible configurations leading to the observation of zero signals, one signal, etc., till $m-1$, where $m$ is the least number of signals required for triggering. In their explicit form, the terms look like the products of the corresponding probability density functions, $p$ for the probability to detect a signal and $\bar{p}=1-p$ for the probability of the non-detection, the index indicates the particular antenna

$$
\begin{aligned}
p^{(1)}= & p_{1} \bar{p}_{2} \cdots \bar{p}_{n-1} \bar{p}_{n}+ \\
& \bar{p}_{1} p_{2} \cdots \bar{p}_{n-1} \bar{p}_{n}+\cdots+ \\
& \bar{p}_{1} \bar{p}_{2} \cdots \bar{p}_{n-1} p_{n} .
\end{aligned}
$$

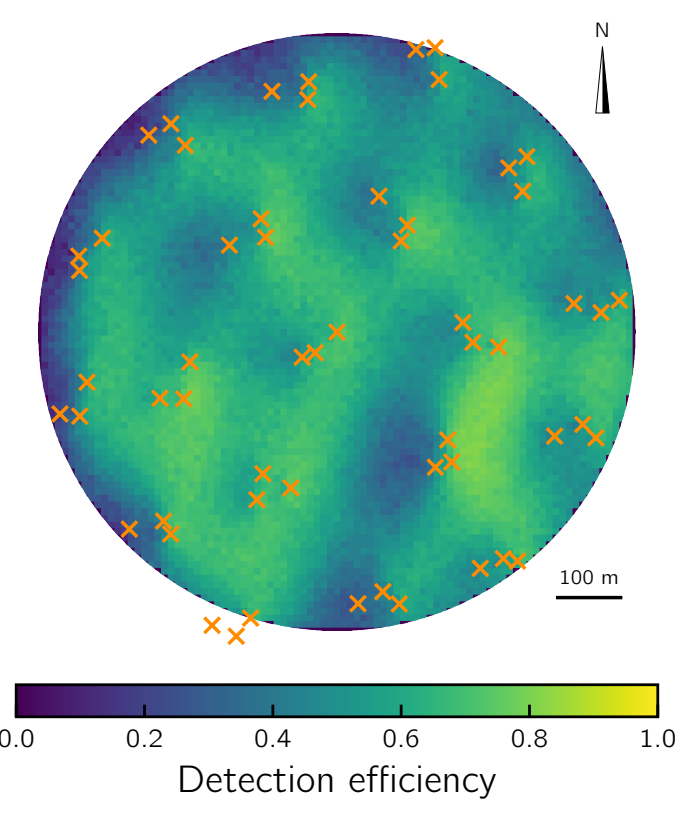

Figure 2: The detection efficiency as a function of the air-shower core location for a shower with the following parameters $\theta=35^{\circ}, \phi=270^{\circ}$, $\log _{10}(E / 1 \mathrm{eV})=17.3, \mathrm{X}_{\max }=658 \mathrm{~g} / \mathrm{cm}^{2}$. The outer circle depicts the border of the instrument fiducial area. The sign in the upper right corner shows the direction towards the geographic north.

This equation gives an example for the probability where only a single antenna has a signal. Each term in the sum is a possible combination of the antennas with and without signals. The total number of the terms equals the total number of the possible combinations described by the corresponding binomial coefficient. In this particular case it is $\left(\begin{array}{c}N \\ 1\end{array}\right)$.

A numerical procedure estimates the probability functions in practical calculations. The peak, the most probable value, of the obtained probability density function is the detection probability for a given air shower. Figure 2 shows the detection probability for multiple air-shower core positions.

This calculation method provides an accurate estimation of the probability density function of the trigger fulfillment. However, its computational complexity rises very fast with the increasing number of antennas required for triggering. The second method, presented below, does not feature such behavior.

Monte-Carlo experiments. Another way to estimate the probability density is to run Monte-Carlo experiments. The idea behind this method is an extension of the existing idea of estimating the trigger probability by running a Bernoulli process for each detector with a given signal-detection probability. In every run, the Bernoulli process on each detector randomly assigns detection or non-detection flag to the detector. By running this procedure multiple times and computing the fractions of configurations leading to satisfying the trigger condition, one can estimate the detection 

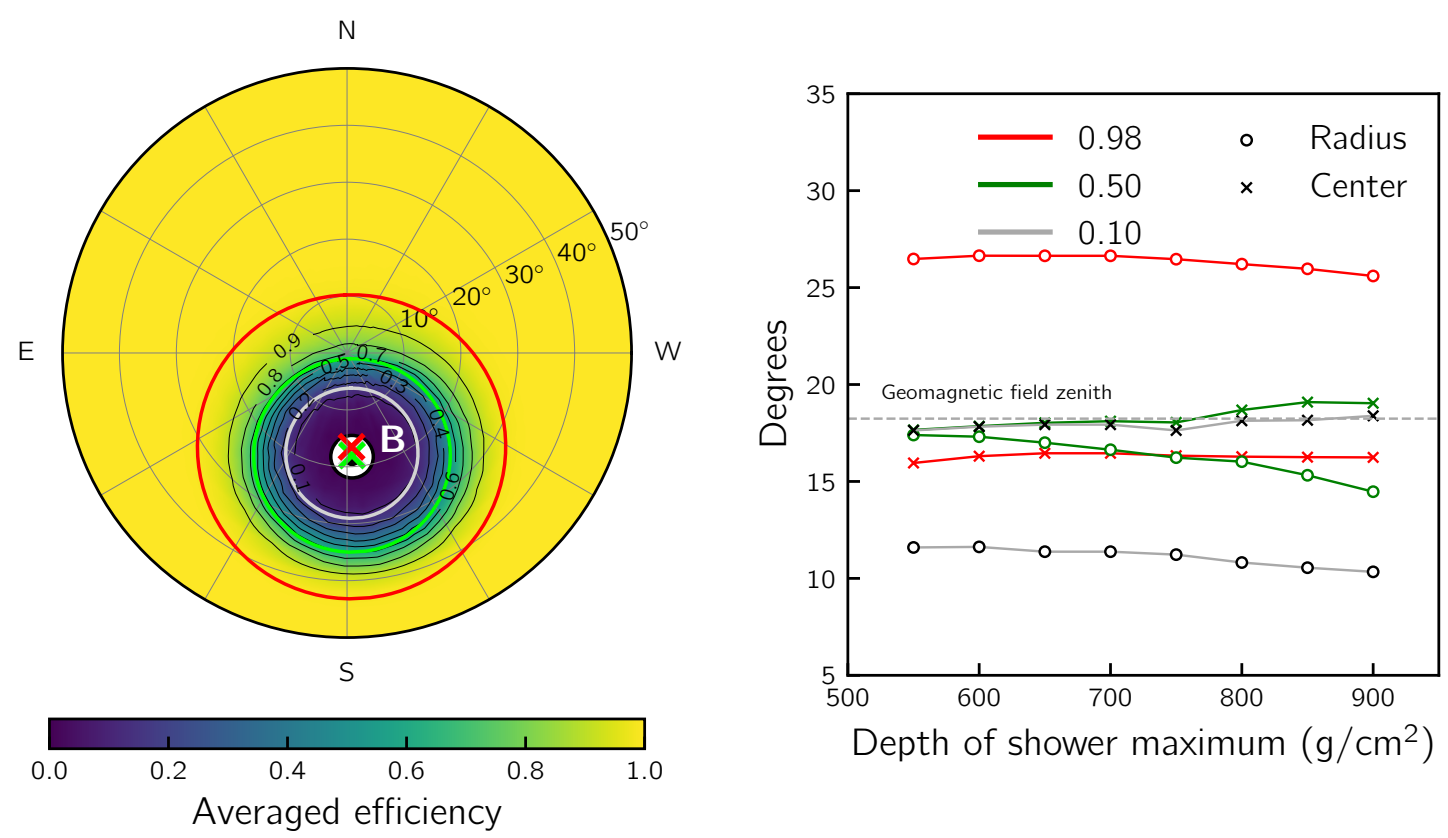

Figure 3: The angular behavior of the averaged efficiency. Left: the distribution of the averaged efficiency over the sky. The red, green, and gray circles correspond to the $0.98,0.5$, and 0.1 maximal efficiency regions. Right: the evolution of the radii and center positions of the circles corresponding to the $0.98,0.50$, and 0.1 maximal efficiency regions. The size of the 0.98 efficiency circle is almost independent of $\mathrm{X}_{\max }$.

probability for an array. In the most simple case, described here, the trigger condition is simply the number of detectors with signals.

Our new method extends this idea from the probabilities to the probability densities. We draw one random sample value from each probability density to detect a signal by an individual antenna and run the procedure described above to obtain a value of the trigger probability. Then the procedure repeats for different sample values of the probability densities. The obtained sample of the trigger probabilities forms the probability density function. The most probable value of the obtained function is the array trigger probability for a given air shower.

\section{Estimation of Aperture}

According to the definition of the angular aperture introduced in the equation (3), its value is the projected averaged efficiency integrated over the sky. The averaged efficiency features quite complex angular behavior, directly impacting the aperture estimation for a radio array. Figure 3 shows the averaged efficiency for Tunka-Rex resulting from the efficiency model described above. As expected from the physics of air-shower radio emission, the efficiency has a suppressed region around the direction of the geomagnetic field.

\subsection{Selection of Full-Efficiency Region}

Even though the behavior of the detection efficiency over the sky is complex, the selection of only the full-efficiency part can be made relatively simple. The region of the suppressed efficiency 
close to the geomagnetic field direction can be approximated with a circle on a sphere. The size of the circular region covering the suppressed efficiency region almost does not depend on the $\mathrm{X}_{\max }$. Figure 3 shows the circular region parameters obtained for a given air-shower energy of $10^{17.3} \mathrm{eV}$ and several positions of $X_{\max }$ ranging from 550 to $900 \mathrm{~g} / \mathrm{cm}^{2}$. As one can see the sizes of the $98 \%$ and $10 \%$ efficiency regions effectively remain the same, however, the size of the $50 \%$ efficiency region undergo significant change. The behavior of the later region is likely connected to the increase of the brightness of the shower while closing the distance to the observer. The exact explanation of the overall angular behavior of the efficiency will be investigated.

\subsection{Evaluation of the Aperture Integral}

It is possible to evaluate the aperture integral within the full efficiency region semianalytically. Namely, express the twodimensional integral in a one-dimensional analytical form, which, in turn, can be computed numerically with very high precision.

The starting point for the integration is to put the averaged efficiency, $\langle\xi\rangle_{s}$, equal to unity. For convenience, we use the spherical coordinate system where the integral appears in its usual form

$$
\begin{aligned}
A_{\Omega}= & \int_{\Omega_{f}}\langle\xi\rangle_{s} \cos \theta d o= \\
& \int_{0}^{2 \pi} \int_{0}^{\theta_{\max }} \cos \theta \sin \theta d \theta d \phi .
\end{aligned}
$$

The whole-sky area within the maximal

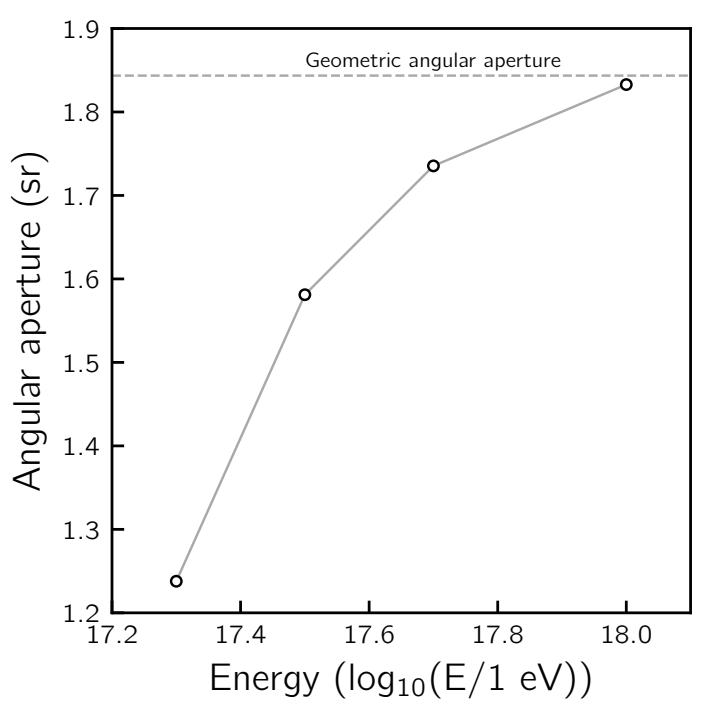

Figure 4: The angular aperture for Tunka-Rex array in the full configuration of 63 antennas and the default trigger condition, at least three antennas with signals. $\left(\right.$ Reference $\mathrm{X}_{\text {max }}=650 \mathrm{~g} / \mathrm{cm}^{2}$.) zenith angle is referred to as region I. The region within the circular area with lower efficiency is referred to as region II, the area within the red circle in Figure 3. The integral is evaluated for all directions within region I, and then the value of the integral for region II is subtracted. The solutions for these two integrals are known, the details of the derivation are in Reference [6]. The solution for the aperture has the form

$$
\begin{aligned}
A_{\Omega}=(I)-(I I)=\pi\left[1-\cos ^{2} \theta_{\text {max }}\right] & - \\
& 2 \int_{0}^{\theta_{\text {max }}} \arccos \left(\frac{\cos \rho-\cos \theta \cos \theta_{0}}{\sin \theta \sin \theta_{0}}\right) \cos \theta \sin \theta d \theta,
\end{aligned}
$$

where the letter $\theta_{0}$ denotes the zenith location of the region II, the letter $\rho$ denotes the angular radius of region II. Factor 2 appears due to the symmetry of the circular region.

Figure 4 shows the estimation of aperture for discrete energies in the range from $10^{17.3}$ to $10^{18.0} \mathrm{eV}$. The size of the suppressed-efficiency region shrinks at the highest energies, making the instrumental aperture almost equal to the geometrical aperture. 


\section{Conclusions and Outlook}

The presented model of the aperture estimation combines two major components: the probabilistic model of the detection efficiency and the semi-analytical approach to the aperture integral estimation. The model incorporates all known uncertainties and uses all available information about the underlying probability distributions. The model's accuracy limitations come only from the accuracy of the suppressed region exclusion and the accuracy of the numerical integration.

With this model it is possible to study the energy and $X_{\max }$ dependence of the efficiency suppression region. Figure 3 shows only the first study of this kind, indicating that the size and location of the efficiency-suppressed region are almost constant over a wide range of $X_{\max }$.

The model has a generic nature and can be used in a wide range of applications. It can be adopted to other air-shower radio arrays with appropriate selection of the footprint description and an update of the signal detection probability.

\section{Acknowledgment}

The present work on the Tunka-Rex project is funded by Deutscher Akademischer Austauschdienst e.V. (personal grant, ref. number 91657437, program ID 57299294). In preparation of this work we used calculations performed on the computational resource ForHLR II funded by the Ministry of Science, Research and the Arts Baden-Württemberg and DFG ("Deutsche Forschungsgemeinschaft"). A part of the data analysis was performed using the radio extension of the Offline framework developed by the Pierre Auger Collaboration [7].

\section{References}

[1] Tunka-Rex Collaboration, P. A. Bezyazeekov et al. Nuclear Instruments and Methods in Physics Research A 802 (Dec., 2015) 89-96.

[2] TAIGA Collaboration, D. Kostunin et al., "Tunka Advanced Instrument for cosmic rays and Gamma Astronomy," in Journal of Physics Conference Series, vol. 1263 of Journal of Physics Conference Series, p. 012006. June, 2019. arXiv: 1903.07460 [astro-ph. IM].

[3] Pierre Auger Collaboration, P. Abreu et al. Journal of Instrumentation 7 no. 10, (Oct., 2012) P10011.

[4] Tunka-Rex Collaboration, V. Lenok et al., "Modeling the Aperture of Radio Instruments for Air-Shower Detection," in 36th International Cosmic Ray Conference (ICRC2019), vol. 36 of International Cosmic Ray Conference, p. 331. July, 2019. arXiv: 1909.01945 [astro-ph.IM].

[5] Tunka-Rex Collaboration, P. A. Bezyazeekov et al. JCAP 01 (2016) 052.

[6] Tunka-Rex Collaboration, V. Lenok et al., "Current Status and New Challenges of The Tunka Radio Extension," in Journal of Physics Conference Series, vol. 1181 of Journal of Physics Conference Series, p. 012027. Feb., 2019. arXiv: 1812.06893 [astro-ph.IM].

[7] Pierre Auger Collaboration, P. Abreu et al. Nucl. Instrum. Meth. A 635 (2011) 92-102. 


\section{Full Authors List: Tunka-Rex Collaboration}

P. Bezyazeekov ${ }^{1}$, N. Budnev ${ }^{1}$, O. Fedorov ${ }^{1}$, O. Gress ${ }^{1}$, O. Grishin ${ }^{1}$, A. Haungs ${ }^{2}$, T. Huege ${ }^{2,3}$, Y. Kazarina ${ }^{1}$, M. Kleifges ${ }^{4}$, E. Korosteleva ${ }^{5}$, D. Kostunin ${ }^{6}$, L. Kuzmichev ${ }^{5}$, V. Lenok ${ }^{2}$, N. Lubsandorzhiev ${ }^{5}$, S. Malakhov ${ }^{1}$, T. Marshalkina ${ }^{1}$, R. Monkhoev ${ }^{1}$, E. Osipova ${ }^{5}$, A. Pakhorukov ${ }^{1}$, L. Pankov ${ }^{1}$, V. Prosin ${ }^{5}$, F. G. Schröder ${ }^{2,7}$ D. Shipilov ${ }^{8}$ and A. Zagorodnikov ${ }^{1}$

${ }^{1}$ Applied Physics Institute ISU, Irkutsk, 664020 Russia

${ }^{2}$ Karlsruhe Institute of Technology, Institute for Astroparticle Physics, D-76021 Karlsruhe, Germany

${ }^{3}$ Astrophysical Institute, Vrije Universiteit Brussel, Pleinlaan 2, 1050 Brussels, Belgium

${ }^{4}$ Institut für Prozessdatenverarbeitung und Elektronik, Karlsruhe Institute of Technology (KIT), Karlsruhe, 76021 Germany

${ }^{5}$ Skobeltsyn Institute of Nuclear Physics MSU, Moscow, 119991 Russia

${ }^{6}$ DESY, Zeuthen, 15738 Germany

${ }^{7}$ Bartol Research Institute, Department of Physics and Astronomy, University of Delaware, Newark, DE, 19716, USA

${ }^{8}$ X5 Retail Group, Moscow, 119049 Russia 